\title{
State stabilization for gate-model quantum computers
}

\author{
Laszlo Gyongyosi ${ }^{1,2,3} \cdot$ Sandor Imre ${ }^{2}$
}

Received: 3 May 2019 / Accepted: 23 July 2019 / Published online: 30 July 2019

(c) The Author(s) 2019

\begin{abstract}
Gate-model quantum computers can allow quantum computations in near-term implementations. The stabilization of an optimal quantum state of a quantum computer is a challenge, since it requires stable quantum evolutions via a precise calibration of the unitaries. Here, we propose a method for the stabilization of an optimal quantum state of a quantum computer through an arbitrary number of running sequences. The optimal state of the quantum computer is set to maximize an objective function of an arbitrary problem fed into the quantum computer. We also propose a procedure to classify the stabilized quantum states of the quantum computer into stability classes. The results are convenient for gate-model quantum computations and near-term quantum computers.
\end{abstract}

Keywords Quantum computers · Gate-model quantum computers ·

Quantum computations · Optimization

\section{Introduction}

Quantum computers can make possible quantum computations for efficient problem solving [1-19]. Gate-based quantum computations represent a way to construct gate-model quantum computers. In a gate-model quantum computer architecture, computations are implemented via sequences of unitary operations [9-12,19-27]. Gate-model quantum computers allow establishing experimental quantum computations in near-term architectures [28-40]. Practical demonstrations of gate-model quantum computers have been already proposed [1-12], and several physical-layer developments are currently in progress.

$凶$ Laszlo Gyongyosi

gyongyosi@hit.bme.hu

1 School of Electronics and Computer Science, University of Southampton, Southampton SO17 1BJ, UK

2 Department of Networked Systems and Services, Budapest University of Technology and Economics, Budapest 1117, Hungary

3 MTA-BME Information Systems Research Group, Hungarian Academy of Sciences, Budapest 1051, Hungary 
Finding a stable quantum state of a quantum computer is a challenge, since it requires precise unitaries that yield stable quantum evolutions in the quantum computer. The problem is further increased if the stable system state must be available for a pre-determined time or for a pre-determined number of running sequences. Particularly, the quantum state of a quantum computer subject to stabilization also coincides with the optimal quantum state. The optimal quantum state of a quantum computer maximizes a particular objective function of an arbitrary computational problem fed into the quantum computer. The problem therefore is to fix the quantum state of the quantum computer in the optimal state for an arbitrary number of running sequences that is determined by the actual environment or by the current problem. Another challenge connected to the problem of stabilization of the system state of a quantum computer is the classification of the sequences of the stabilized quantum states into stability classes. Practically, a solution to these problems can be covered by an unsupervised learning method.

Here, we propose a method for the stabilization of an optimal quantum state of a quantum computer through an arbitrary number of running sequences. We define a solution that utilizes unsupervised learning algorithms to determine the stable quantum states of the quantum computer and to classify the stable quantum states into stability classes. The proposed results are useful for experimental gate-based quantum computations and near-term quantum computer architectures.

The novel contributions of our manuscript are as follows:

1. We propose a method for the stabilization of an optimal quantum state of a quantum computer through an arbitrary number of running sequences.

2. We define a solution that utilizes unsupervised learning algorithms to determine the stable quantum states of the quantum computer.

3. We evaluate a solution to classify the stable system states into stability classes.

This paper is organized as follows. Section 2 provides the problem statement. Section 3 discusses the stabilization procedure of an optimal quantum state of a quantum computer. Section 4 defines an unsupervised learning method to find the stable quantum states and the stability classes of the stabilized quantum states. In Sect. 5, a numerical evaluation is proposed. Finally, Sect. 6 concludes with the results. Supplemental information is included in the Appendix.

\section{Problem statement}

Let $Q G$ be the quantum gate structure of a gate-model quantum computer with a sequence of $L$ unitaries [9-12] with an $n$-length input system $|\psi\rangle$,

$$
|\psi\rangle=\sum_{i=0}^{d^{n}-1} \alpha_{i}|i\rangle
$$

where $d$ is the dimension ( $d=2$ for a qubit system), $\sum_{i=0}^{d^{n}-1}\left|\alpha_{i}\right|^{2}=1$, and let

$$
\left|\vec{\theta}^{*}\right\rangle=U_{L}\left(\theta_{L}^{*}\right) U_{L-1}\left(\theta_{L-1}^{*}\right) \ldots U_{1}\left(\theta_{1}^{*}\right)|\psi\rangle
$$


be the optimal system state of the quantum computer that maximizes a particular objective function $f\left(\vec{\theta}^{*}\right)$,

$$
f\left(\vec{\theta}^{*}\right)=\left\langle\vec{\theta}^{*}|C| \vec{\theta}^{*}\right\rangle
$$

of an arbitrary problem fed into the quantum computer, where $C$ is the classical value of the objective function, while $\vec{\theta}^{*}$ is the gate parameter vector,

$$
\vec{\theta}^{*}=\left[\theta_{1}^{*}, \ldots, \theta_{L}^{*}\right]^{T}
$$

that identifies the $L$ unitaries, $U_{1}\left(\theta_{1}^{*}\right), \ldots, U_{1}\left(\theta_{L}^{*}\right)$, of the $Q G$ quantum circuit of the quantum computer in the optimal state $\left|\vec{\theta}^{*}\right\rangle$, such that an $i$-th unitary, $U_{i}\left(\theta_{i}^{*}\right)$ is as [10]

$$
U_{i}\left(\theta_{i}^{*}\right)=\exp \left(-i \theta_{i}^{*} P\right)
$$

where $\theta_{i}^{*}$ is the gate parameter (real continuous variable) of unitary $U_{i}, P$ is a generalized Pauli operator formulated by the tensor product of Pauli operators $\{X, Y, Z\}$ $[10,11]$.

The aim is to stabilize the $\left|\vec{\theta}^{*}\right\rangle$ optimal state of the quantum computer through $R$ running sequences via unsupervised learning of the evolution of the unitaries in the quantum computer.

The $R$ running sequences refer to $R$ input systems fed into the input of the quantum computer, such that in an $r$-th running sequence, $r=1, \ldots, R$, an $r$-th input system, $\left|\psi_{r}\right\rangle$ (defined as in (1)), is evolved via the sequence of the $L$ uniaries of the quantum computer. The $R$ running sequences identify an input system, $\left|\psi_{i n}\right\rangle$, formulated via $R, n$-length quantum systems, as

$$
\left|\psi_{i n}\right\rangle=\left|\psi_{1}\right\rangle \otimes \cdots \otimes\left|\psi_{R}\right\rangle
$$

where it is considered that the $R$ input systems are unentangled.

Let $\vec{\varphi}$ be the gate parameter vector associated with the stable system state $|\vec{\varphi}\rangle$,

$$
|\vec{\varphi}\rangle=U_{L}\left(\varphi_{L}\right) U_{L-1}\left(\varphi_{L-1}\right) \ldots U_{1}\left(\varphi_{1}\right)|\psi\rangle
$$

as

$$
\vec{\varphi}=\left[\varphi_{1}, \ldots, \varphi_{L}\right]^{T}
$$

where $\varphi_{i} \in[0, \pi]$ is the gate parameter of unitary $U_{i}$ in the stabilized system state $|\vec{\varphi}\rangle$, such that the objective function value is stabilized into

$$
f(\vec{\varphi})=\langle\vec{\varphi}|C| \vec{\varphi}\rangle=f\left(\vec{\theta}^{*}\right)
$$


For the $R$ sequences of the quantum computer, we define matrices $\alpha$ and $\beta$ as

$$
\alpha=\left[\vec{\theta}_{1}^{*}, \ldots, \vec{\theta}_{R}^{*}\right]
$$

where $\vec{\theta}_{r}^{*}=\left[\theta_{r, 1}^{*}, \ldots, \theta_{r, L}^{*}\right]^{T}$ identifies the quantum state $\left|\vec{\theta}_{r}^{*}\right\rangle$ of an $r$-th running sequence of the quantum computer, while

$$
\beta=\left[\vec{\varphi}_{1}, \ldots, \vec{\varphi}_{R}\right]
$$

where $\vec{\varphi}_{r}=\left[\varphi_{r, 1}, \ldots, \varphi_{r, L}\right]^{T}$, identifies the stabilized quantum state $\left|\vec{\varphi}_{r}\right\rangle$ of an $r$-th sequence of the quantum computer.

The problem therefore is to find $\beta$ from $\alpha$ that stabilizes the $\left|\vec{\theta}^{*}\right\rangle$ optimal state of the quantum computer through $R$ sequences as

$$
\beta=S^{T} \alpha,
$$

where $S$ is a stabilizer matrix,

$$
S^{T} S=I,
$$

and $I$ is the identity matrix.

The problems to be solved are therefore summarized as follows.

Problem 1 Find $S$ to construct $\beta$ (11) from $\alpha$ (10) to stabilize the quantum computer in $\left|\vec{\theta}^{*}\right\rangle$ via $|\vec{\varphi}\rangle$ for all running sequences.

Problem 2 Describe the stability of $\beta$ via unsupervised learning of the stability levels of the $\left|\vec{\varphi}_{r}\right\rangle$ quantum states of $\beta$.

The resolutions of Problems 1 and 2 are proposed in Theorems 1 and 2 . The solution framework $\mathcal{F}$ is defined via a $\mathcal{P}_{S}$ stabilization procedure with an embedded stabilization algorithm $\mathcal{A}_{S}$ (see Theorem 1), and via an $\mathcal{A}_{C}$ classification algorithm that characterizes the stability class of the results of $\mathcal{P}_{S}$ (see Theorem 2). Figure 1 depicts the system model.

\section{Stabilization of the optimal state of the quantum computer}

Theorem 1 The $S$ matrix for the stabilization of the $\left|\vec{\theta}^{*}\right\rangle$ optimal state of the quantum computer via $\beta=S^{T} \alpha$ can be determined via the minimization of an objective function $F^{*}$. 


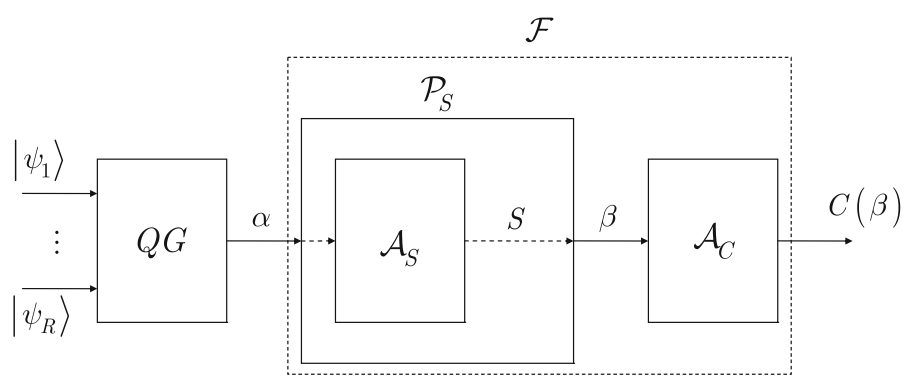

Fig. 1 The framework $\mathcal{F}$ for the stabilization of the optimal state of the quantum computer and the stability class determination. In the $R$ running sequences, $R$ input systems are fed into the input of the quantum computer, in an $r$-th running sequence, $r=1, \ldots, R$, an $r$-th input system, $\left|\psi_{r}\right\rangle=\sum_{i} \alpha_{i}|i\rangle$, is evolved via the sequence of the $L$ uniaries of the quantum computer. The $R$ running sequences identify an input system $\left|\psi_{i n}\right\rangle=\left|\psi_{1}\right\rangle \otimes \cdots \otimes\left|\psi_{R}\right\rangle$ (considering that the $R$ input systems are unentangled). The $R$ running sequences of the $Q G$ structure of the quantum computer produces $\alpha=\left[\vec{\theta}_{1}^{*}, \ldots, \vec{\theta}_{R}^{*}\right]$, where $\vec{\theta}_{r}^{*}=\left[\theta_{r, 1}^{*}, \ldots, \theta_{r, L}^{*}\right]^{T}$. The $\mathcal{P}_{S}$ stabilization procedure outputs $\beta=\left[\vec{\varphi}_{1}, \ldots, \vec{\varphi}_{R}\right]$, where $\vec{\varphi}_{r}=\left[\varphi_{r, 1}, \ldots, \varphi_{r, L}\right]^{T}$, via an embedded stabilization algorithm $\mathcal{A}_{S}$ that determines the $S$ stabilizer matrix. The $C(\beta)$ stability level of the resulting $\beta$ is determined via a classification algorithm $\mathcal{A}_{C}$. The $\mathcal{P}_{S}$ and $\mathcal{A}_{S}$ methods are realized as unsupervised learning

Proof For an $r$-th sequence of the quantum computer, define $\Delta\left(\vec{\theta}_{r}^{*}\right)$ and $\Delta\left(\vec{\varphi}_{r}\right)$ as

$$
\Delta\left(\vec{\theta}_{r}^{*}\right)=\vec{\theta}_{r}^{*}-\vec{\theta}_{r+1}^{*}
$$

and

$$
\Delta\left(\vec{\varphi}_{r}\right)=\vec{\varphi}_{r}-\vec{\varphi}_{r+1},
$$

respectively. These vectors formulate $\Delta \alpha$ and $\Delta \beta$ as

$$
\Delta \alpha=\left[\Delta\left(\vec{\theta}_{1}^{*}\right), \ldots, \Delta\left(\vec{\theta}_{R-1}^{*}\right)\right]
$$

and

$$
\Delta \beta=\left[\Delta\left(\vec{\varphi}_{1}\right), \ldots, \Delta\left(\vec{\varphi}_{R-1}\right)\right],
$$

respectively. Then, using Eqs. (16) and (17) for the $r=1, \ldots, R-1$ sequences, let $\chi$ be a sum defined as

$$
\begin{aligned}
\chi & =\sum_{r}^{R-1}\left\|\Delta\left(\vec{\varphi}_{r}\right)\right\|_{2}^{2} \\
& =\operatorname{Tr}\left(\Delta \beta(\Delta \beta)^{T}\right) \\
& =\operatorname{Tr}\left(S^{T}\left(\Delta \alpha(\Delta \alpha)^{T}\right) S\right),
\end{aligned}
$$


where $\|\cdot\|_{2}^{2}$ is the squared L2-norm, $\operatorname{Tr}(\cdot)$ is the trace operator, $\Delta \alpha$ is as given in Eq. (16), and $\Delta \beta$ is as in Eq. (17).

For the $r$-th and $s$-th sequences, $s>r$, with $\Delta\left(\vec{\varphi}_{r}\right)$ and $\Delta\left(\vec{\varphi}_{s}\right)$, let $\gamma_{r s}$ be defined as

$$
\gamma_{r s}=\omega_{r s}\left\|\Delta\left(\vec{\varphi}_{r}\right)-\Delta\left(\vec{\varphi}_{s}\right)\right\|_{2}^{2},
$$

where $\omega_{r s}$ is a weight coefficient defined as

$$
\omega_{r s}=\left\{\begin{array}{l}
\exp \left(-\frac{\left\|\Delta\left(\vec{\theta}_{r}^{*}\right)-\Delta\left(\vec{\theta}_{s}^{*}\right)\right\|^{2}}{\zeta}\right), \text { if }(s-r) \leq \kappa \\
0, \text { otherwise }
\end{array}\right.
$$

where $\kappa$ and $\zeta$ are nonzero parameters.

A sum is defined for the $r=1, \ldots, R-1$ sequences of the quantum computer as

$$
\tau=\sum_{r}^{R-1} \sum_{s}^{R-1} \gamma_{r s} .
$$

At a particular $S$ in Eqs. (18) and (21), the stabilization of the optimal state of the quantum computer through the $R$ sequences can be reformulated via an objective function $F^{*}$, subject to a minimization as

$$
\begin{aligned}
F^{*} & =\arg \min _{S}(\chi+c \tau) \\
& =\arg \min _{S}\left(\operatorname{Tr}\left(S^{T}\left(\Delta \alpha(\Delta \alpha)^{T}\right) S\right)+c \tau\right),
\end{aligned}
$$

where $c$ is a regularization constant [41,42]. The $F^{*}$ objective function therefore stabilizes the optimal state via the minimization of $\chi$, while the term $c \tau$ achieves stabilization between the sequences.

Then, let $W$ be the weight matrix formulated via coefficients (20) with $W_{r s}=\omega_{r s}$, and let $\eta$ be a diagonal matrix of the weight coefficients (20) with

$$
\eta_{r r}=\sum_{s} \omega_{r s}
$$

such that

$$
(\Delta \beta)^{T} \eta \Delta \beta=I
$$


Using $W$ and $\eta$, the $F^{*}$ objective function in Eq. (22) can be rewritten as

$$
\begin{aligned}
F^{*} & =\arg \min _{S}\left(\frac{1}{\Omega}\left(\operatorname{Tr}\left(\Delta \beta(\Delta \beta)^{T}\right)+c \operatorname{Tr}\left(\Delta \beta(\eta-W)(\Delta \beta)^{T}\right)\right)\right) \\
& =\arg \min _{S}\left(\frac{1}{\Omega}\left(\operatorname{Tr}\left(\Delta \beta(I+c(\eta-W))(\Delta \beta)^{T}\right)\right)\right) \\
& =\arg \min _{S}\left(\frac{1}{\Omega}\left(\operatorname{Tr}\left(\Delta \beta \sigma(\Delta \beta)^{T}\right)\right)\right) \\
& =\arg \min _{S}\left(\frac{1}{\Omega}\left(\operatorname{Tr}\left(S^{T}\left(\Delta \alpha \sigma(\Delta \alpha)^{T}\right) S\right)\right)\right)
\end{aligned}
$$

where $\sigma$ is as

$$
\sigma=I+c(\eta-W)
$$

and

$$
\Omega=\operatorname{Tr}\left(S^{T}\left(\Delta \alpha \eta(\Delta \alpha)^{T}\right) S\right)
$$

At a particular $\Delta \alpha(16)$ and $\sigma$ (26), the $S$ stabilizer matrix in Eq. (25) is evaluated via

$$
\left(\Delta \alpha \sigma(\Delta \alpha)^{T}\right) S=\lambda\left(\Delta \alpha \eta(\Delta \alpha)^{T}\right) S
$$

where $\lambda$ is a diagonal matrix of eigenvalues [41,42].

Algorithm A.1 $\left(\mathcal{A}_{S}\right)$ gives the method for stabilizing the optimal state of the quantum computer.

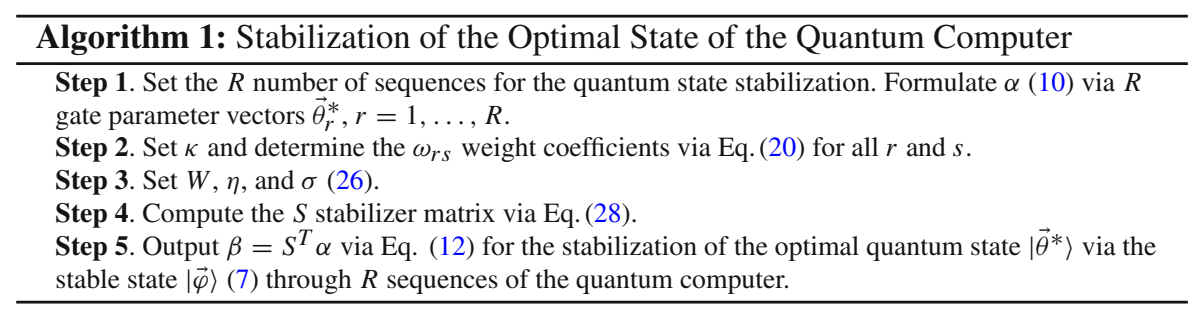

\section{Learning the stable quantum state and stability class}

Lemma 1 The stabilized sequences of the quantum computer can be determined via unsupervised learning. 
Proof Algorithm 1 with the objective function (25) can be used to formulate an unsupervised learning framework to find the stabilized unitaries. The steps are detailed in Procedure $1\left(\mathcal{P}_{S}\right)$.

\section{Procedure 1: Unsupervised Learning of Stable Quantum Evolutions}

Step 1. Construct a $\mathcal{T}$ training set of random gate parameters of the $Q G$-structure of the quantum computer, as

$$
\mathcal{T}=\left(X_{1}, \ldots, X_{q}\right)
$$

where $X_{i}$ is a $K$-dimensional random vector, $d \leq R$, formulated as

$$
X_{i}=\left[\theta_{i, 1}, \ldots, \theta_{i, K}\right]
$$

where $\theta_{i, j}$ is the gate parameter of $U_{j}$ in $X_{i}$, and $j$ is a random number.

Step 2. Determine the $S$ stabilizer matrix via Algorithm 1.

Step 3. Compute $Z=\left[z_{1}, \ldots, z_{q}\right]$ as

$$
Z=\left(S^{T} \mathcal{T}^{T}\right)^{T}=\mathcal{T} S
$$

and set $B=\left[b_{1}, \ldots, b_{q}\right]$ as

$$
B=-Z^{T} \overline{\mathcal{T}}
$$

where $\overline{\mathcal{T}}$ is the mean of all training samples [42].

Step 4. For a given $\vec{\theta}_{r}^{*}$ of an $r$-th sequence, learn output $Y_{r}$ as

$$
Y_{r}=Z^{T} \vec{\theta}_{r}^{*}+B=(\mathcal{T} S)^{T} \vec{\theta}_{r}^{*}-(\mathcal{T} S)^{T} \overline{\mathcal{T}}
$$

Step 5. For an $i$-th gate parameter $\theta_{r, i}^{*}$, learn the $j$-th output $y_{i, j}^{(r)}$ as

$$
y_{i, j}^{(r)}=z_{j} \otimes \theta_{r, i}^{*}+b_{j}
$$

from which a statistical average for a given $i, i=1, \ldots, L$, is

$$
\tilde{y}_{i}^{(r)}=\frac{1}{q} \sum_{j=1}^{q}\left|y_{i, j}^{(r)}\right|,
$$

with difference $\Delta \tilde{y}_{i}^{(r)}$ as

$$
\Delta \tilde{y}_{i}^{(r)}=\left|\tilde{y}_{i}^{(r)}-\tilde{y}_{i+1}^{(r)}\right| .
$$

Step 6. Repeat step 5 for all $i$.

Step 7. Repeat steps 1-5 for the $R$ sequences. 


\subsection{Learning the sequence stability of stabilized quantum states}

Proposition 1 The stability of a given sequence $\vec{\varphi}_{r}$ can be characterized via $K$ stability levels. The sequence $\vec{\varphi}_{r}$ can be classified into $K$ stability classes from set $\mathcal{C}$,

$$
\mathcal{C}=\left\{C_{1}, \ldots, C_{K}\right\}
$$

where $C_{k}, k=1, \ldots, K$, is the $k$-th stability class.

Theorem 2 The $C\left(\vec{\varphi}_{r}\right), r=1, \ldots, R$ stability class of a $\vec{\varphi}_{r}$ stabilized sequence, $\vec{\varphi}_{r}=\varphi_{r, 1}, \ldots, \varphi_{r, L}$, of the quantum computer can be learned via $\phi_{k}\left(\vec{\varphi}_{r}\right)=$ $\left(v_{k}\left(\vec{\varphi}_{r}\right)\right)^{T} f_{k}^{\mathcal{C}}\left(\vec{\varphi}_{r}\right)$ quantities in the high-dimensional Hilbert space $\mathcal{H}$, where $v_{k}\left(\varphi_{r, i}\right)=\frac{1}{\pi}\left(\varphi_{r, i}\right)$, and $f_{k}^{\mathcal{C}}\left(\varphi_{r, i}\right) \in[0,1]$ is a probability.

Proof Since the gate parameters are stabilized, the gate parameters $\vec{\varphi}_{r}$ and $\vec{\varphi}_{r+1}$ of the $r$-th and $(r+1)$-th sequences must be correlated in the stable system state $|\vec{\varphi}\rangle(7)$ of the quantum computer.

Let $\beta$ from Eq. (11) be the $R$ stabilized sequences, where $\vec{\varphi}_{r}$ is the stabilized gate parameter vector of an $r$-th sequence of the quantum computer, and let $\mathcal{S}$ be the set of all sequences of gate parameters as

$$
\mathcal{S}=\bigcup_{\vec{\varphi}_{r} \in \beta}\left\{\varphi_{r, i} \mid \varphi_{r, i} \in \vec{\varphi}_{r}\right\} .
$$

For an $k$-th stabilization class $C_{k}$, a probabilistic classifier function $f_{k}^{\mathcal{C}}[41,43]$ can be defined as

$$
f_{k}^{\mathcal{C}}: \mathcal{S} \rightarrow[0,1]
$$

The goal is to learn a function that maps any $\vec{\varphi}_{r}$ sequence to the correct stability class. Applying Eq. (39) on a given sequence $\vec{\varphi}_{r}$, i.e., $f_{k}^{\mathcal{C}}\left(\vec{\varphi}_{r}\right)$ therefore maps $\vec{\varphi}_{r}$ to a given stability class via the classification of each $L$ gate parameter of the sequence.

Thus, an $i$-th stabilized gate parameter $\varphi_{r, i}$ of an $r$-th sequence $\vec{\varphi}_{r}$ can be also classified into a particular stabilization class from $\mathcal{C}(37)$. The $f_{k}^{\mathcal{C}}\left(\varphi_{r, i}\right) \in[0,1]$, $k=1, \ldots, K$, classifier (39) is trained to classify [43] each of the $\varphi_{r, i}$ gate parameters of $\vec{\varphi}_{r}, i=1, \ldots, L$ via outputting a corresponding probability that $\varphi_{r, i}$ belongs to a given $C_{k}$ class. For a particular $\varphi_{r, i}$, the sum of the probabilities yields

$$
\sum_{k=1}^{K} f_{k}^{\mathcal{C}}\left(\varphi_{r, i}\right)=1,
$$

for all $i$.

Then, let $v_{k}\left(\varphi_{r, i}\right) \geq 0$ be a weight parameter associated with a particular $\varphi_{r, i}$ and $k$-th class $C_{k}$, defined as

$$
v_{k}\left(\varphi_{r, i}\right)=\frac{1}{\pi}\left(\varphi_{r, i}\right)
$$

which normalizes $\varphi_{r, i}$ into the range of $[0,1], v_{k}\left(\varphi_{r, i}\right) \in[0,1]$. 
For an $r$-th sequence $\vec{\varphi}_{r}$, a $v_{k}\left(\vec{\varphi}_{r}\right)$ collection can be defined as

$$
v_{k}\left(\vec{\varphi}_{r}\right)=\left[v_{k}\left(\varphi_{r, 1}\right), \ldots, v_{k}\left(\varphi_{r, L}\right)\right],
$$

where $\sum_{i=1}^{L} v_{k}\left(\varphi_{r, i}\right)=1$.

From Eqs. (39) and (42), the $\phi_{k}\left(\vec{\varphi}_{r}\right)$ evolution of a particular sequence $\vec{\varphi}_{r}$ with respect to a $k$-th class $C_{k}$ is defined as

$$
\begin{aligned}
\phi_{k}\left(\vec{\varphi}_{r}\right) & =\left(v_{k}\left(\vec{\varphi}_{r}\right)\right)^{T} f_{k}^{\mathcal{C}}\left(\vec{\varphi}_{r}\right) \\
& =\left[v_{k}\left(\varphi_{r, 1}\right) f_{k}^{\mathcal{C}}\left(\varphi_{r, 1}\right), \ldots, v_{k}\left(\varphi_{r, L}\right) f_{k}^{\mathcal{C}}\left(\varphi_{r, L}\right)\right] .
\end{aligned}
$$

Since the $\phi_{k}\left(\vec{\varphi}_{r}\right)$ term (43) is a nonlinear map, the problem of correlation analysis $[41,43]$ between the inner products of nonlinear functions $\phi_{k}\left(\vec{\varphi}_{r}\right)$ and $\phi_{l}\left(\vec{\varphi}_{r}\right)$ can be reformulated via a kernel machine $\mathcal{K}[44-46]$ as $\mathcal{K}\left(\phi_{k}\left(\vec{\varphi}_{r}\right), \phi_{l}\left(\vec{\varphi}_{r}\right)\right)$, which yields a distance in a high-dimensional Hilbert space $\mathcal{H}$. This distance in $\mathcal{H}$ can therefore be used as a metric to describe the correlation between $\phi_{k}\left(\vec{\varphi}_{r}\right)$ and $\phi_{l}\left(\vec{\varphi}_{r}\right)$.

Let $\mathcal{X}$ be the input space, and let $\mathcal{K}$ be an arbitrary kernel machine, defined for a given $x, y \in \mathcal{X}$ via the kernel function

$$
\mathcal{K}(x, y)=\Gamma(x)^{T} \Gamma(y),
$$

where

$$
\Gamma: \mathcal{X} \rightarrow \mathcal{H}
$$

is a nonlinear map from $\mathcal{X}$ to the high-dimensional reproducing kernel Hilbert space (RKHS) $\mathcal{H}$ associated with $\mathcal{K}$. Without a loss of generality, $\operatorname{dim}(\mathcal{H}) \gg \operatorname{dim}(\mathcal{X})$, and we assume that the map $\Gamma$ in Eq. (45) has no inverse.

Then, for a $\phi_{k}\left(\vec{\varphi}_{r}\right)$ and $\phi_{l}\left(\vec{\varphi}_{r}\right)$, let $\rho\left(\phi_{k}\left(\vec{\varphi}_{r}\right), \phi_{l}\left(\vec{\varphi}_{r}\right)\right) \rightarrow \mathcal{H}$ be the correlation identifier, as

$$
\begin{aligned}
& \rho\left(\phi_{k}\left(\vec{\varphi}_{r}\right), \phi_{l}\left(\vec{\varphi}_{r}\right)\right) \\
& =\mathcal{K}\left(\phi_{k}\left(\vec{\varphi}_{r}\right), \phi_{l}\left(\vec{\varphi}_{r}\right)\right) \\
& =\sum_{i=1}^{L} \mathcal{K}\left(v_{k}\left(\varphi_{r, i}\right) f_{k}^{\mathcal{C}}\left(\varphi_{r, i}\right), v_{l}\left(\varphi_{r, i}\right) f_{l}^{\mathcal{C}}\left(\varphi_{r, i}\right)\right) .
\end{aligned}
$$

Assuming that $\mathcal{K}$ is a Gaussian kernel [44-46] in Eq. (46), for an $i$-th gate parameter the kernel function is

$$
\begin{aligned}
\mathcal{K} & \left(v_{k}\left(\varphi_{r, i}\right) f_{k}^{\mathcal{C}}\left(\varphi_{r, i}\right), v_{l}\left(\varphi_{r, i}\right) f_{l}^{\mathcal{C}}\left(\varphi_{r, i}\right)\right) \\
& =\exp \left(-\frac{1}{c} f_{d}\left(v_{k}\left(\varphi_{r, i}\right) f_{k}^{\mathcal{C}}\left(\varphi_{r, i}\right), v_{l}\left(\varphi_{r, i}\right) f_{l}^{\mathcal{C}}\left(\varphi_{r, i}\right)\right)\right),
\end{aligned}
$$

where $c=2 \sigma^{2}$, while $f_{d}(\cdot)$ yields the L2-distance in $\mathcal{H}$,

$$
\begin{aligned}
& f_{d}\left(v_{k}\left(\varphi_{r, i}\right) f_{k}^{\mathcal{C}}\left(\varphi_{r, i}\right), v_{l}\left(\varphi_{r, i}\right) f_{l}^{\mathcal{C}}\left(\varphi_{r, i}\right)\right) \\
&=\left\|v_{k}\left(\varphi_{r, i}\right) f_{k}^{\mathcal{C}}\left(\varphi_{r, i}\right)-v_{l}\left(\varphi_{r, i}\right) f_{l}^{\mathcal{C}}\left(\varphi_{r, i}\right)\right\|_{2}^{2} .
\end{aligned}
$$


For a given $\phi_{k}\left(\vec{\varphi}_{r}\right)$ and $\phi_{l}\left(\vec{\varphi}_{r}\right)$, an $f_{A}\left(\phi_{c}\left(\vec{\varphi}_{r}\right), \phi_{c}\left(\vec{\varphi}_{r}\right)\right) \rightarrow \delta_{+}^{K}$ average is yielded as

$$
\begin{aligned}
& \varsigma\left(\phi_{c}\left(\vec{\varphi}_{r}\right), \phi_{c}\left(\vec{\varphi}_{r}\right)\right)=\left(\phi_{c}\left(\vec{\varphi}_{r}\right)\right)^{T} \phi_{c}\left(\vec{\varphi}_{r}\right) \\
& =\sum_{i=1}^{L} v_{c}^{2}\left(\varphi_{r, i}\right)\left(f_{c}^{\mathcal{C}}\left(\varphi_{r, i}\right)\right)^{2} \leq \sum_{i=1}^{L} v_{c}\left(\varphi_{r, i}\right) f_{c}^{\mathcal{C}}\left(\varphi_{r, i}\right),
\end{aligned}
$$

where $\delta_{+}^{K}$ refers to the space of $K \times K$ symmetric positive semi-definite matrices $[43,45,46]$, while the $\iota$ inner products of $\phi_{k}\left(\vec{\varphi}_{r}\right)$ and $\phi_{l}\left(\vec{\varphi}_{r}\right)$ are represented in $\delta_{+}^{K}$ via $\iota\left(\phi_{k}\left(\vec{\varphi}_{r}\right), \phi_{l}\left(\vec{\varphi}_{r}\right)\right) \rightarrow \delta_{+}^{K}$ as

$$
\begin{aligned}
& \iota\left(\phi_{k}\left(\vec{\varphi}_{r}\right), \phi_{l}\left(\vec{\varphi}_{r}\right)\right)=\left(\phi_{k}\left(\vec{\varphi}_{r}\right)\right)^{T} \phi_{l}\left(\vec{\varphi}_{r}\right) \\
& \quad=\sum_{i=1}^{L} v_{k}\left(\varphi_{r, i}\right) v_{l}\left(\varphi_{r, i}\right)\left(f_{k}^{\mathcal{C}}\left(\varphi_{r, i}\right)\right)\left(f_{l}^{\mathcal{C}}\left(\varphi_{r, i}\right)\right) .
\end{aligned}
$$

The $\vec{\varphi}_{r}$ sequence is classified into a given class from set $\mathcal{C}$, as given in Algorithm 2 $\left(\mathcal{A}_{C}\right)$.

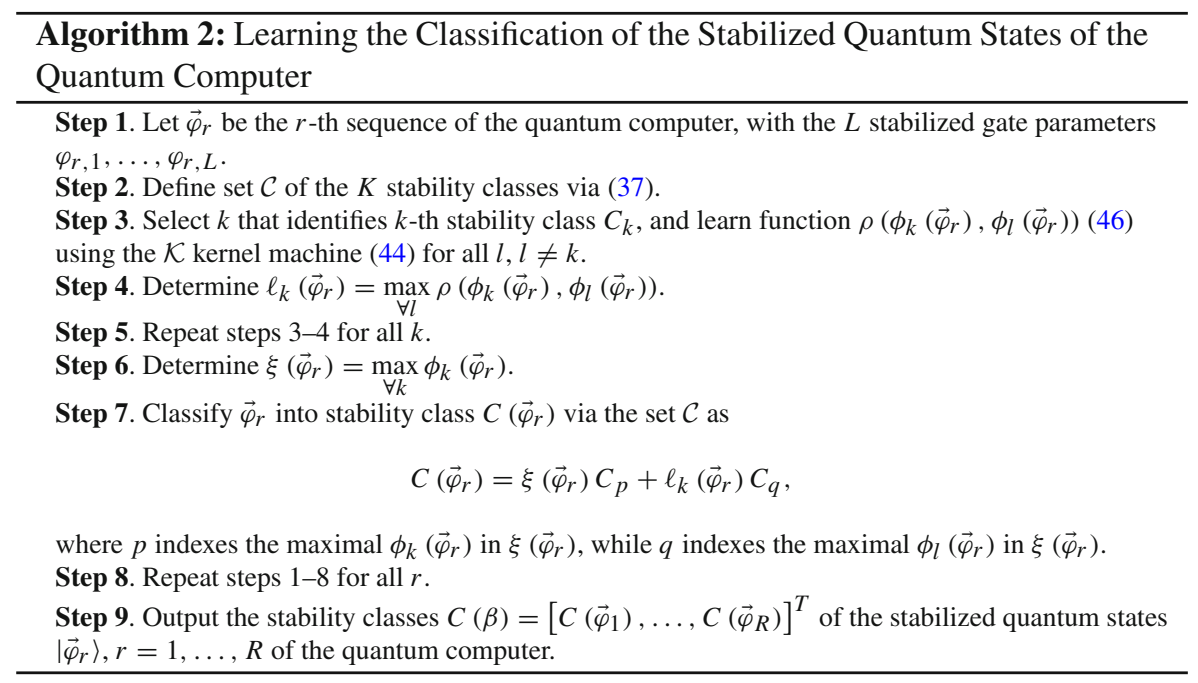

\section{Numerical evaluation}

\subsection{System stability}

Let $|\phi\rangle$ be the stabilized system state of the quantum computer formulated by $R$ output systems, $\left|\vec{\varphi}_{r}\right\rangle, r=1, \ldots, R$, as 


$$
|\phi\rangle=\left|\vec{\varphi}_{1}\right\rangle \otimes \cdots \otimes\left|\vec{\varphi}_{R}\right\rangle
$$

with gate parameters $\beta$, as given in (11).

Then, let $\left|\phi^{*}\right\rangle$ be a target stabilized system of the quantum computer, as

$$
\left|\phi^{*}\right\rangle=\left|\vec{\varphi}_{1}^{*}\right\rangle \otimes \cdots \otimes\left|\vec{\varphi}_{R}^{*}\right\rangle
$$

with target gate parameters $\beta^{*}$, as

$$
\beta^{*}=\left[\vec{\varphi}_{1}^{*}, \ldots, \vec{\varphi}_{R}^{*}\right]
$$

where $\vec{\varphi}_{r}^{*}=\left[\varphi_{r, 1}^{*}, \ldots, \varphi_{r, L}^{*}\right]^{T}$.

Then, let $[\beta]_{r l}$ refer to the gate parameter $\varphi_{r, l}$ of an $l$-th unitary of an $r$-th running sequence of the quantum computer, $l=1, \ldots, L, r=1, \ldots, R$, in the state $|\phi\rangle$, and let $\left[\beta^{*}\right]_{r l}$ identify the target gate parameter $\varphi_{r, l}^{*}$ in state $\left|\phi^{*}\right\rangle$.

Then, let $D\left(\beta \| \beta^{*}\right)$ be the relative entropy between $\beta$ and $\beta^{*}$, as

$$
D\left(\beta \| \beta^{*}\right)=\sum_{r, l}\left([\beta]_{r l} \log \frac{[\beta]_{r l}}{\left[\beta^{*}\right]_{r l}}+\left[\beta^{*}\right]_{r l}-[\beta]_{r l}\right) .
$$

where $D\left(\beta \| \beta^{*}\right) \geq 0$, and let $f_{D\left(\beta \| \beta^{*}\right)}(r) \geq 0$ be a function that returns the value of the relative entropy function for an $r$-th running sequence as

$$
f_{D\left(\beta \| \beta^{*}\right)}(r)=\sum_{l}\left([\beta]_{r l} \log \frac{[\beta]_{r l}}{\left[\beta^{*}\right]_{r l}}+\left[\beta^{*}\right]_{r l}-[\beta]_{r l}\right) .
$$

Let $f_{D\left(\beta \| \beta^{*}\right)}^{*}(r)$ be a target value for function (55), and let $\Delta\left(f_{D\left(\beta \| \beta^{*}\right)}(r)\right)$ be the difference [47] between $f_{D\left(\beta \| \beta^{*}\right)}^{*}(r)$ and (55), as

$$
\Delta\left(f_{D\left(\beta \| \beta^{*}\right)}(r)\right)=\frac{1}{R} \int_{1}^{R} \partial_{D\left(\beta \| \beta^{*}\right)}^{2}(r) \mathrm{d} r,
$$

where $\partial_{D\left(\beta \| \beta^{*}\right)}(r)$ is the derivative of $f_{D\left(\beta \| \beta^{*}\right)}(r)$.

Using (56), we define a stability parameter $\delta$ to quantify the variation of the $\left|\vec{\varphi}_{r}\right\rangle$ stabilized system state of the $r$-th running sequence of the quantum computer, as

$$
\delta(r)=\left(\frac{R}{2 \pi} \sqrt{\Delta\left(f_{D\left(\beta \| \beta^{*}\right)}(r)\right)}\right)^{-1} .
$$

For analytical purposes, let us assume that $f_{D\left(\beta \| \beta^{*}\right)}(r)$ oscillates between a minimal value $\gamma \geq 0$, and a maximal value $\gamma \leq \lambda \leq 1$, defined as

$$
\gamma=\arg \min _{\forall r}\left(f_{D\left(\beta \| \beta^{*}\right)}(r)\right),
$$


and

$$
\lambda=\arg \max _{\forall r}\left(f_{D\left(\beta \| \beta^{*}\right)}(r)\right),
$$

therefore $f_{D\left(\beta \| \beta^{*}\right)}(r)$ can be rewritten as

$$
f_{D\left(\beta \| \beta^{*}\right)}(r)=c \sin \left(N 2 \pi \frac{r}{R}\right)+\mathbb{E}\left(D\left(\beta \| \beta^{*}\right)\right),
$$

where $c$ is a constant, set as

$$
c=\frac{1}{2}(\lambda-\gamma)
$$

while $0 \leq \mathbb{E}\left(D\left(\beta \| \beta^{*}\right)\right) \leq 1$ is an expected value of (54), set as

$$
\mathbb{E}\left(D\left(\beta \| \beta^{*}\right)\right)=c+\gamma
$$

while $N$ is the number of oscillations.

Therefore, (56) can be evaluated as

$$
\begin{aligned}
\Delta\left(f_{D\left(\beta \| \beta^{*}\right)}(r)\right) & =\frac{1}{R} \int_{1}^{R} \frac{2 N^{2} 4 \pi^{2}}{R^{2}} \cos ^{2}\left(N 2 \pi \frac{r}{R}\right) \mathrm{d} r \\
& =\frac{2 N^{2} 4 \pi^{2}}{R^{2}} \frac{1}{N 2 \pi} \int_{1}^{N 2 \pi} 2 \cos ^{2}\left(r^{\prime}\right) d r^{\prime} \\
& =\frac{N^{2} 4 \pi^{2}}{R^{2}},
\end{aligned}
$$

where $r \in\left[r_{0}, r_{0}+R\right]$, with $r_{0}=1$.

Then, by using (63), the quantity in (57) is as

$$
\delta(r)=\left(\frac{R}{2 \pi} \sqrt{\frac{N^{2} 4 \pi^{2}}{R^{2}}}\right)^{-1}=\frac{1}{N},
$$

that identifies the inverse of the number of oscillations.

Therefore, (64) identifies the stability of the system state $\left|\vec{\varphi}_{r}\right\rangle$ of the quantum computer in the $r$-th running sequence if $f_{D\left(\beta \| \beta^{*}\right)}(r)$ has the form of (60). For an arbitrary $f_{D\left(\beta \| \beta^{*}\right)}(r)$, the stability parameter $\delta(r)$ is evaluated via (57). The high value of $\delta(r)$ indicates that the stabilized system $\left|\vec{\varphi}_{r}\right\rangle$ in (51) changes slowly. Particularly, if $\delta(r) \geq \delta^{*}(r)$, where $\delta^{*}(r)$ is a target value for $\delta(r)$, then the system state $\left|\vec{\varphi}_{r}\right\rangle$ of the quantum computer is considered as stable.

The values of $f_{D\left(\beta \| \beta^{*}\right)}(r)(60)$ and $\delta(r)$ (64) for $R$ running sequences are depicted in Fig. 2. 
(a)

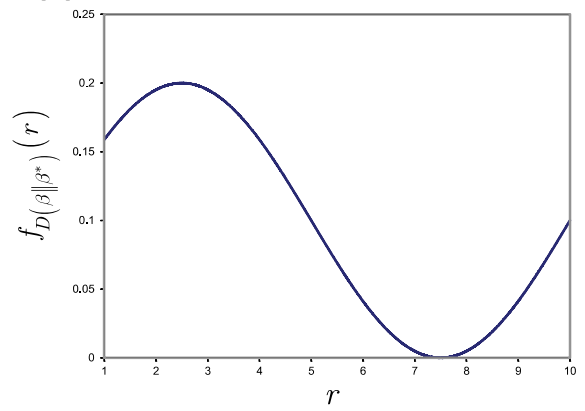

(c)

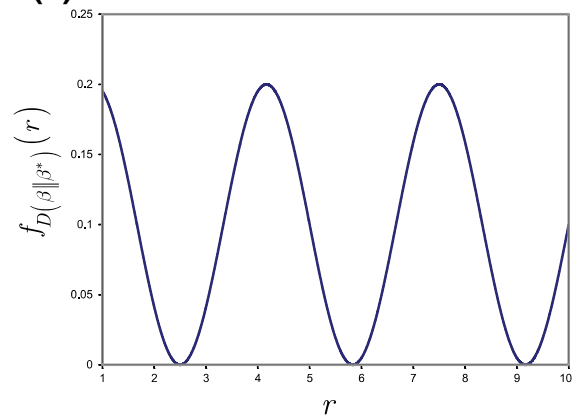

(b)

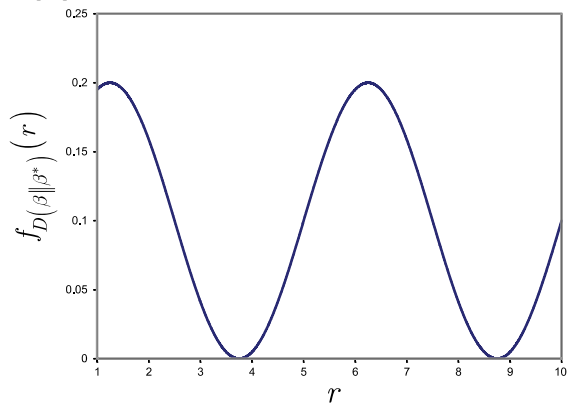

(d)

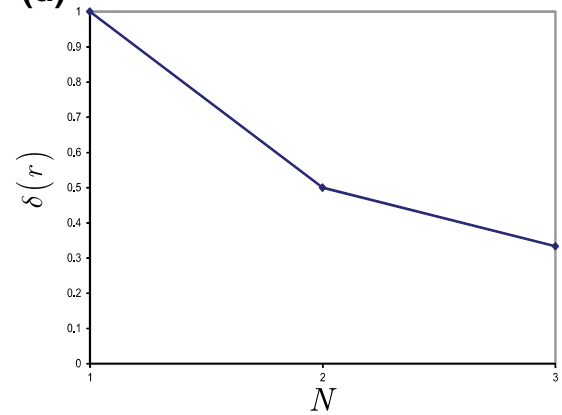

Fig. 2 The $f_{D\left(\beta \| \beta^{*}\right)}(r)$ relative entropy values between the gate parameters of the stabilized system $\left|\vec{\varphi}_{r}\right\rangle$ and target system $\left|\vec{\varphi}_{r}^{*}\right\rangle$ for $R$ running sequences, $r=1, \ldots R, R=10, \mathbb{E}\left(D\left(\beta \| \beta^{*}\right)\right)=0.1$. a $N=1$. b $N=2$. c $N=3$. d Stability parameter $\delta(r)$ for the different relative entropy values

\subsection{Gate parameter correlations}

Let $\left|\vec{\varphi}_{r}\right\rangle$ be the stabilized state of the quantum computer in the $r$-th running sequence, with $\vec{\varphi}_{r}=\left[\varphi_{r, 1}, \ldots, \varphi_{r, L}\right]^{T}$, and let $\left|\vec{\varphi}_{r}^{*}\right\rangle$ be the target stabilized system state in the $r$-th running sequence, with $\vec{\varphi}_{r}^{*}=\left[\varphi_{r, 1}^{*}, \ldots, \varphi_{r, L}^{*}\right]^{T}$.

Then, let $\mu$ be a correlation coefficient [47] that measures the correlation of the gate parameters $\beta$ and $\beta^{*}$ of $|\phi\rangle(51)$ and $\left|\phi^{*}\right\rangle(11)$, defined as

$$
\mu\left(\beta, \beta^{*}\right)=\left|\frac{F\left(\left(f\left(\vec{\varphi}_{r}\right)-F\left(f\left(\vec{\varphi}_{r}\right)\right)\right)\left(f\left(\vec{\varphi}_{r}^{*}\right)-F\left(f\left(\vec{\varphi}_{r}^{*}\right)\right)\right)\right)}{\sqrt{F\left(\left(f\left(\vec{\varphi}_{r}\right)-F\left(f\left(\vec{\varphi}_{r}\right)\right)\right)^{2}\right) F\left(\left(f\left(\vec{\varphi}_{r}^{*}\right)-F\left(f\left(\vec{\varphi}_{r}^{*}\right)\right)\right)^{2}\right)}}\right|,
$$

where $|\cdot|$ is the absolute value, $f\left(\vec{\varphi}_{r}\right)$ is a function of $r$ that represents the values of the gate parameter vector $\vec{\varphi}_{r}$, while $F(\cdot)$ is defined over $r \in[1, R]$, as

$$
F(f(x))=\frac{1}{R} \int_{1}^{R} f(x) \mathrm{d} r .
$$


For illustration purposes, let us assume that $L=1$, and $f\left(\vec{\varphi}_{r}\right)$ is as

$$
f\left(\vec{\varphi}_{r}\right)=X \cos ^{2}\left(C N 2 \pi \frac{r}{R}\right)
$$

where we set $X$ as $X=2 C^{2} N^{2} 4 \pi^{2} / R^{2}$, while $C>0$ is a constant, thus (66) is evaluated as

$$
\begin{aligned}
F\left(f\left(\vec{\varphi}_{r}\right)\right) & =\frac{1}{R} \int_{1}^{R} \frac{2 C^{2} N^{2} 4 \pi^{2}}{R^{2}} \cos ^{2}\left(C N 2 \pi \frac{r}{R}\right) \mathrm{d} r \\
& =\frac{2 C^{2} N^{2} 4 \pi^{2}}{R^{2}} \frac{1}{N 2 \pi} \int_{1}^{N 2 \pi} 2 \cos ^{2}\left(r^{\prime}\right) d r^{\prime} \\
& =\frac{C^{2} N^{2} 4 \pi^{2}}{R^{2}} .
\end{aligned}
$$

For the target system $\left|\vec{\varphi}_{r}^{*}\right\rangle$, the constant $C^{*}$ is set as

$$
f\left(\vec{\varphi}_{r}^{*}\right)=\frac{2\left(C^{*}\right)^{2} N^{2} 4 \pi^{2}}{R^{2}} \cos ^{2}\left(C^{*} N 2 \pi \frac{r}{R}\right)
$$

while for $\left|\vec{\varphi}_{r}\right\rangle$, we set $C$ as $C>C^{*}$, thus (65) can be evaluated as

$$
\mu\left(\beta, \beta^{*}\right)=\left|\frac{F\left(\left(f\left(\vec{\varphi}_{r}\right)-\frac{C^{2} N^{2} 4 \pi^{2}}{R^{2}}\right)\left(f\left(\vec{\varphi}_{r}^{*}\right)-\frac{\left(C^{*}\right)^{2} N^{2} 4 \pi^{2}}{R^{2}}\right)\right)}{\sqrt{F\left(\left(f\left(\vec{\varphi}_{r}\right)-\frac{C^{2} N^{2} 4 \pi^{2}}{R^{2}}\right)^{2}\right) F\left(\left(f\left(\vec{\varphi}_{r}^{*}\right)-\frac{\left(C^{*}\right)^{2} N^{2} 4 \pi^{2}}{R^{2}}\right)^{2}\right)}}\right|,
$$

where

$$
\begin{aligned}
& F\left(\left(f\left(\vec{\varphi}_{r}\right)-\frac{C^{2} N^{2} 4 \pi^{2}}{R^{2}}\right)\left(f\left(\vec{\varphi}_{r}^{*}\right)-\frac{\left(C^{*}\right)^{2} N^{2} 4 \pi^{2}}{R^{2}}\right)\right) \\
& \quad=F\left(f\left(\vec{\varphi}_{r}\right) f\left(\vec{\varphi}_{r}^{*}\right)-f\left(\vec{\varphi}_{r}\right) \frac{\left(C^{*}\right)^{2} N^{2} 4 \pi^{2}}{R^{2}}-\frac{C^{2} N^{2} 4 \pi^{2}}{R^{2}} f\left(\vec{\varphi}_{r}^{*}\right)+\frac{C^{2} N^{2} 4 \pi^{2}}{R^{2}} \frac{\left(C^{*}\right)^{2} N^{2} 4 \pi^{2}}{R^{2}}\right) \\
& \quad=\frac{1}{R}\left(\frac{2 \pi^{3} C^{2}\left(C^{*}\right)^{2} N^{3}\left(\left(C^{*}-C\right) \sin \left(N 4 \pi\left(C^{*}+C\right)\right)+\left(C^{*}+C\right) \sin \left(N 4 \pi\left(C^{*}-C\right)\right)\right)}{\left(\left(C^{*}\right)^{2}-C^{2}\right) R^{3}}\right)
\end{aligned}
$$

and

$$
\begin{aligned}
F\left(\left(f\left(\vec{\varphi}_{r}\right)-\frac{C^{2} N^{2} 4 \pi^{2}}{R^{2}}\right)^{2}\right) & =F\left(f\left(\vec{\varphi}_{r}\right)^{2}-2 f\left(\vec{\varphi}_{r}\right) \frac{C^{2} N^{2} 4 \pi^{2}}{R^{2}}+\left(\frac{C^{2} N^{2} 4 \pi^{2}}{R^{2}}\right)^{2}\right) \\
& =\frac{1}{R}\left(\frac{C^{4} N^{4} 8 \pi^{4}}{R^{3}}\right)=\frac{C^{4} N^{4} 8 \pi^{4}}{R^{4}},
\end{aligned}
$$


(a)

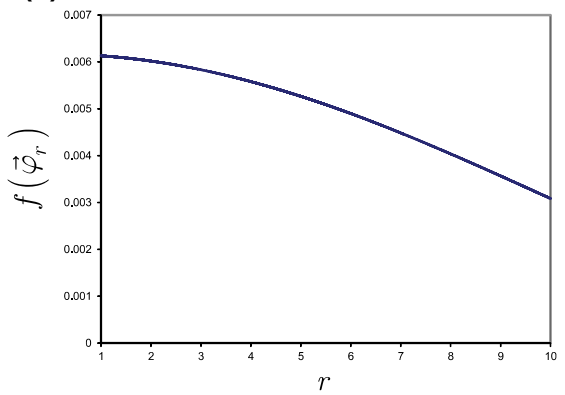

(c)

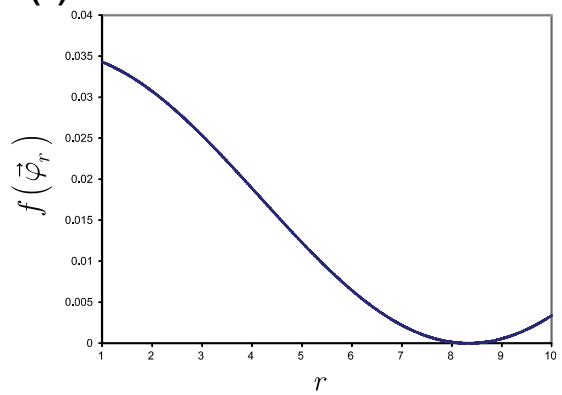

(e)

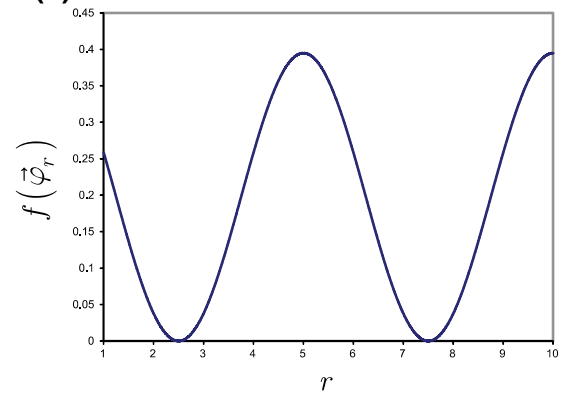

(b)

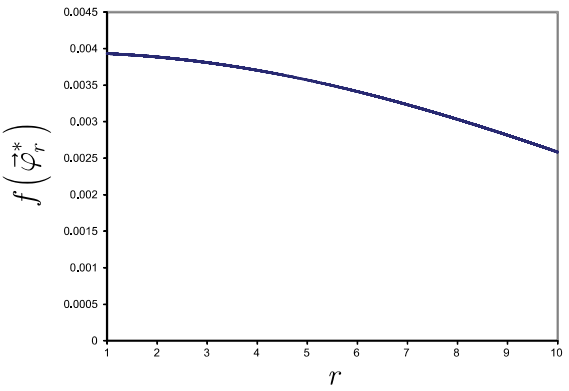

(d)

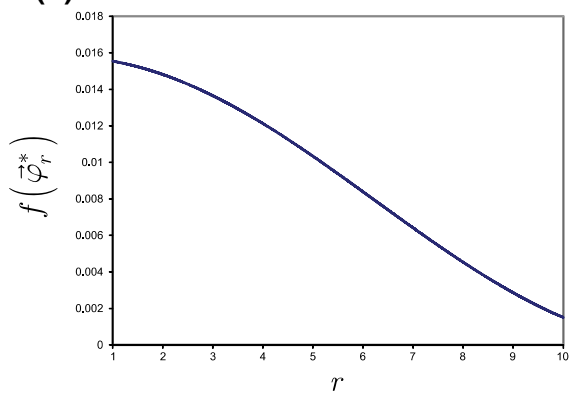

(f)

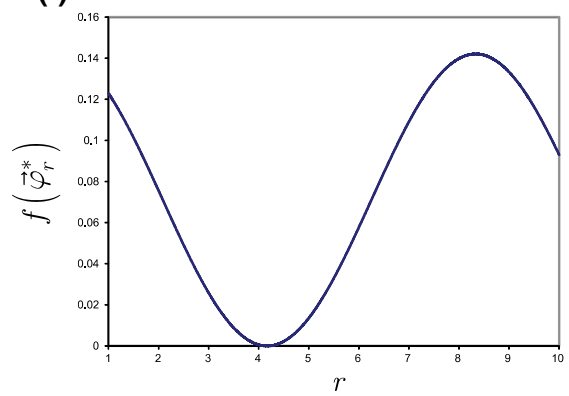

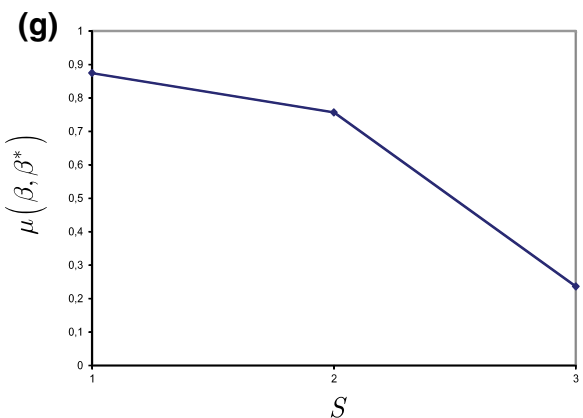

Fig. 3 Gate parameter values of $f\left(\vec{\varphi}_{r}\right)$ and $f\left(\vec{\varphi}_{r}^{*}\right)$ in function of running sequence $r, r=1, \ldots, R$, $R=10, L=1$, for different $N, C$ and $C^{*}, C \in[0,1], C^{*} \in[0,1] . \mathbf{a}, \mathbf{b} N=1, C=0.125, C^{*}=0.1$. c, d $N=1, C=0.3, C^{*}=0.2 \mathbf{e}, \mathbf{f} N=2, C=0.5, C^{*}=0.3$. $\mathbf{g}$ The $\mu\left(\beta, \beta^{*}\right)$ correlation values between the gate parameters of $\mathbf{a}-\mathbf{f}, S$ is an indexing parameter 

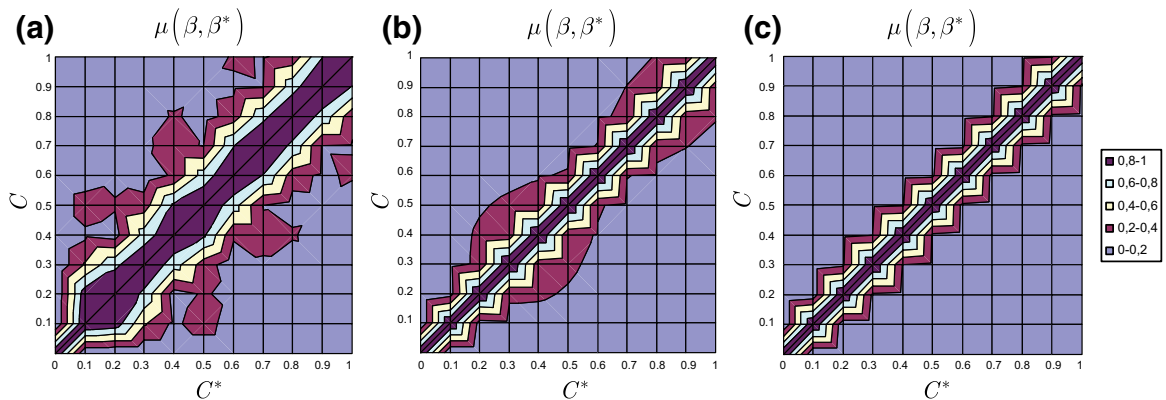

Fig. 4 The distribution of $\mu\left(\beta, \beta^{*}\right)$ in function of $C$ and $C^{*}$ at $f\left(\vec{\varphi}_{r}\right)$ and $f\left(\vec{\varphi}_{r}^{*}\right), L=1, R=10$. a $N=1$. $\mathbf{b} N=2$. $\mathbf{c} N=3$

thus (70) is simplified as

$$
\mu\left(\beta, \beta^{*}\right)=\left|\frac{2 \pi^{3} C^{2}\left(C^{*}\right)^{2} N^{3}\left(\left(C^{*}-C\right) \sin \left(N 4 \pi\left(C^{*}+C\right)\right)+\left(C^{*}+C\right) \sin \left(N 4 \pi\left(C^{*}-C\right)\right)\right)}{\left(\left(\left(C^{*}\right)^{2}-C^{2}\right) R^{4}\right) \sqrt{\frac{\left.C^{4} N^{4}\right)}{R^{4}} \pi^{4}} \frac{\left(C^{*}\right)^{4} N^{4} 8 \pi^{4}}{R^{4}}}\right| .
$$

The values of (73) are depicted in Fig. 3.

In Fig. 4, the distribution of $\mu\left(\beta, \beta^{*}\right)$ in function of $C$ and $C^{*}$ is depicted, $C \in[0,1]$, $C^{*} \in[0,1]$ for different values of $N, f\left(\vec{\varphi}_{r}\right)$ and $f\left(\vec{\varphi}_{r}^{*}\right)$ are evaluated as given in (67) and (69), $L=1$, and $R=10$.

\section{Conclusions}

Here, we defined a method for the learning of stable quantum evolutions in gate-model quantum computer architectures. The model stabilizes an optimal state of a quantum computer to maximize the particular objective function of an arbitrary problem fed into the quantum computer. The model learns a stabilizer matrix that stabilizes the state of the quantum computer through an arbitrary number of run sequences. We also defined a scheme to characterize the stability of the stabilized states via unsupervised learning of the stability classes of the stabilized sequences. The results are particularly useful for gate-based quantum computations and gate-model quantum computer architectures.

Acknowledgements Open access funding provided by Budapest University of Technology and Economics (BME). The research reported in this paper has been supported by the National Research, Development and Innovation Fund (TUDFO/51757/2019-ITM, Thematic Excellence Program). This work was partially supported by the National Research Development and Innovation Office of Hungary (Project No. 20171.2.1-NKP-2017-00001), by the Hungarian Scientific Research Fund - OTKA K-112125 and in part by the BME Artificial Intelligence FIKP grant of EMMI (BME FIKP-MI/SC).

Author contributions L.GY. designed the protocol and wrote the manuscript. L.GY. and S.I. analyzed the results. All authors reviewed the manuscript.

Funding No relevant funding. 


\section{Compliance with ethical standards}

Ethics statement This work did not involve any active collection of human data.

Data accessibility statement This work does not have any experimental data.

Competing financial interests statement We have no competing financial interests.

Competing interests statement We have no competing interests.

Open Access This article is distributed under the terms of the Creative Commons Attribution 4.0 International License (http://creativecommons.org/licenses/by/4.0/), which permits unrestricted use, distribution, and reproduction in any medium, provided you give appropriate credit to the original author(s) and the source, provide a link to the Creative Commons license, and indicate if changes were made.

\section{A Appendix}

\section{A.1 Abbreviations}

QG Quantum Gate structure of a gate-model quantum computer RKHS Reproducing Kernel Hilbert Space

\section{A.2 Notations}

The notations of the manuscript are summarized in Table 1.

Table 1 Summary of notations

\begin{tabular}{ll}
\hline$Q G$ & Quantum gate structure of a gate-model quantum computer \\
$L$ & Number of unitaries in the $Q G$ structure of the quantum computer \\
$U_{i}\left(\theta_{i}\right)$ & An $i$-th unitary gate, $U_{i}\left(\theta_{i}\right)=\exp \left(-i \theta_{i} P\right)$, where $P$ is a generalized Pauli \\
& operator formulated by a tensor product of Pauli operators $\{X, Y, Z\}$, while $\theta_{i}$ is \\
& referred to as the gate parameter associated to $U_{i}\left(\theta_{i}\right)$ \\
$|\vec{\theta}\rangle$ & System state of the quantum computer, $|\vec{\theta}\rangle=U_{L}\left(\theta_{L}\right) U_{L-1}\left(\theta_{L-1}\right) \ldots U_{1}\left(\theta_{1}\right)$, \\
& where $U_{i}\left(\theta_{i}\right)$ identifies an $i$-th unitary gate \\
$\vec{\theta}$ & Gate parameter vector, a collection of gate parameters of the $L$ unitaries, \\
& $\vec{\theta}=\left[\theta_{1}, \ldots, \theta_{L-1}, \theta_{L}\right]^{T}$ \\
$C$ & Classical objective function of a computational problem fed into the quantum \\
& computer \\
$f(\vec{\theta})$ & Objective function of the quantum computer \\
$\left|\vec{\theta}^{*}\right\rangle$ & Optimal state of the quantum computer \\
$\vec{\theta} \vec{\theta}^{*}$ & Gate parameter vector in the $\left|\vec{\theta}^{*}\right\rangle$ system state, $\vec{\theta}^{*}=\left[\theta_{1}^{*}, \ldots, \theta_{L}^{*}\right]^{T}$ \\
$f\left(\vec{\theta}^{*}\right)$ & Objective function value in the $\left|\vec{\theta}^{*}\right\rangle$ system state \\
$P$ & Generalized Pauli operator formulated by the tensor product of Pauli operators \\
$|\vec{\varphi}\rangle$ & $\{X, Y, Z\}$
\end{tabular}


Table 1 continued

\begin{tabular}{|c|c|}
\hline$\vec{\varphi}$ & Gate parameter vector associated to the stable system state $|\vec{\varphi}\rangle, \vec{\varphi}=\left[\varphi_{1}, \ldots, \varphi_{L}\right]^{T}$ \\
\hline$\vec{\theta}_{r}^{*}$ & $\begin{array}{l}\text { Gate parameter vector, identifies the quantum state }\left|\vec{\theta}_{r}^{*}\right\rangle \text { of an } r \text {-th running } \\
\text { sequence, } r=1, \ldots, R \text {, of the quantum computer, } \vec{\theta}_{r}^{*}=\left[\theta_{r, 1}^{*}, \ldots, \theta_{r, L}^{*}\right]^{T}\end{array}$ \\
\hline$\vec{\varphi}_{r}$ & $\begin{array}{l}\text { Gate parameter vector, identifies the stabilized quantum state }\left|\vec{\varphi}_{r}\right\rangle \text { of an } r \text {-th } \\
\text { sequence of the quantum computer, } \vec{\varphi}_{r}=\left[\varphi_{r, 1}, \ldots, \varphi_{r, L}\right]^{T}\end{array}$ \\
\hline$\alpha$ & $\begin{array}{l}\text { Matrix, formulated via the } R \text { sequences of the quantum computer, } \\
\qquad \alpha=\left[\vec{\theta}_{1}^{*}, \ldots, \vec{\theta}_{R}^{*}\right]\end{array}$ \\
\hline$\beta$ & $\begin{array}{l}\text { Matrix, formulated via the } R \text { stabilized sequences of the quantum computer, } \\
\quad \beta=\left[\vec{\varphi}_{1}, \ldots, \vec{\varphi}_{R}\right]\end{array}$ \\
\hline$S$ & $\begin{array}{l}\text { Stabilizer matrix, yields } \beta \text { from } \alpha \text { as } \beta=S^{T} \alpha, S^{T} S=I \text {, where } I \text { is the identity } \\
\text { matrix }\end{array}$ \\
\hline $\mathcal{F}$ & Solution framework \\
\hline $\mathcal{P}_{S}$ & Stabilization procedure \\
\hline $\mathcal{A}_{S}$ & Stabilization algorithm \\
\hline $\mathcal{A}_{C}$ & Classification algorithm \\
\hline$C(\beta)$ & Stability class of $\beta$ \\
\hline$\Delta\left(\vec{\theta}_{r}^{*}\right)$ & Vector, defined for an $r$-th sequence of the quantum computer, $\Delta\left(\vec{\theta}_{r}^{*}\right)=\vec{\theta}_{r}^{*}-\vec{\theta}_{r+1}^{*}$ \\
\hline$\Delta\left(\vec{\varphi}_{r}\right)$ & $\begin{array}{l}\text { Vector, defined for an } r \text {-th stabilized sequence of the quantum computer, } \\
\qquad \Delta\left(\vec{\varphi}_{r}\right)=\vec{\varphi}_{r}-\vec{\varphi}_{r+1}\end{array}$ \\
\hline$\Delta \alpha$ & A collection of $\Delta\left(\vec{\theta}_{r}^{*}\right)$ vectors, $\Delta \alpha=\left[\Delta\left(\vec{\theta}_{1}^{*}\right), \ldots, \Delta\left(\vec{\theta}_{R-1}^{*}\right)\right]$ \\
\hline$\Delta \beta$ & A collection of $\Delta\left(\vec{\varphi}_{r}\right)$ vectors, $\Delta \beta=\left[\Delta\left(\vec{\varphi}_{1}\right), \ldots, \Delta\left(\vec{\varphi}_{R-1}\right)\right]$ \\
\hline$\chi$ & Sum defined via $\Delta \beta$ as $\chi=\sum_{r}^{R-1}\left\|\Delta\left(\vec{\varphi}_{r}\right)\right\|_{2}^{2}$, where $\|\cdot\|_{2}^{2}$ is the squared L2-norm \\
\hline$\gamma_{r s}$ & $\begin{array}{l}\text { Parameter, defined as } \gamma_{r s}=\omega_{r s}\left\|\Delta\left(\vec{\varphi}_{r}\right)-\Delta\left(\vec{\varphi}_{s}\right)\right\|_{2}^{2}, \text { where } \Delta\left(\vec{\varphi}_{r}\right) \text { and } \Delta\left(\vec{\varphi}_{s}\right) \\
\text { are derived for an } r \text {-th and } s \text {-th sequences, } s>r \text {, while } \omega_{r s} \text { is a weight } \\
\text { coefficient }\end{array}$ \\
\hline$\tau$ & $\begin{array}{l}\text { A sum, defined for the } r=1, \ldots, R-1 \text { sequences of the quantum computer, } \\
\tau=\sum_{r}^{R-1} \sum_{s}^{R-1} \gamma_{r s} \text {. }\end{array}$ \\
\hline$F^{*}$ & Objective function of the stabilization procedure \\
\hline$c$ & Regularization constant \\
\hline$\omega_{r s}$ & $\begin{array}{l}\text { Weight coefficient for the } r \text {-th and } s \text {-th sequences, } s>r \text {, } \\
\omega_{r s}=\left\{\begin{array}{l}\exp \left(-\frac{\left\|\Delta\left(\vec{\theta}_{r}^{*}\right)-\Delta\left(\vec{\theta}_{s}^{*}\right)\right\|^{2}}{\zeta}\right), \text { if }(s-r) \leq \kappa \\
0, \text { otherwise }\end{array} \text {, where } \kappa \text { and } \zeta \text { are }\right. \\
\text { nonzero parameters }\end{array}$ \\
\hline$W$ & Weight matrix, $W_{r s}=\omega_{r s}$ \\
\hline$\eta$ & $\begin{array}{l}\text { Diagonal matrix of the weight coefficients, } \eta_{r r}=\sum_{s} \omega_{r s} \text {, with relation } \\
\qquad(\Delta \beta)^{T} \eta \Delta \beta=I\end{array}$ \\
\hline$\sigma$ & Matrix, $\sigma=I+c(\eta-W)$ \\
\hline$\Omega$ & Parameter, $\Omega=\operatorname{Tr}\left(S^{T}\left(\Delta \alpha \eta(\Delta \alpha)^{T}\right) S\right)$ \\
\hline$\lambda$ & Diagonal matrix of eigenvalues \\
\hline $\mathcal{T}$ & $\begin{array}{l}\text { Training set of random gate parameters of the } Q G \text {-structure of the quantum } \\
\text { computer, } \mathcal{T}=\left(X_{1}, \ldots, X_{q}\right) \text {, where } X_{i} \text { is a } d \text {-dimensional random vector }\end{array}$ \\
\hline
\end{tabular}


Table 1 continued

\begin{tabular}{|c|c|}
\hline$\overline{\mathcal{T}}$ & Mean of all training samples \\
\hline$Y_{r}$ & Learned output for an $r$-th sequence \\
\hline$y_{i, j}^{(r)}$ & Learned $j$-th output for an $i$-th unitary of an $r$-th sequence \\
\hline$\Delta \tilde{y}_{i}^{(r)}$ & Difference, $\Delta \tilde{y}_{i}^{(r)}=\left|\tilde{y}_{i}^{(r)}-\tilde{y}_{i+1}^{(r)}\right|$. \\
\hline$C_{k}$ & $\begin{array}{l}\text { A } k \text {-th stability class, } k=1, \ldots, K \text {, for the classification of the stability of the } \\
\text { stabilized sequences of } \beta \text {. }\end{array}$ \\
\hline $\mathcal{C}$ & Set of $K$ stability classes, $\mathcal{C}=\left\{C_{1}, \ldots, C_{K}\right\}$ \\
\hline$C\left(\vec{\varphi}_{r}\right)$ & Stability class of a stabilized sequence $\vec{\varphi}_{r}$ \\
\hline$C(\beta)$ & $\begin{array}{l}\text { Stability classes of all } \vec{\varphi}_{r} \text { stabilized sequences, } r=1, \ldots, R \text {, of } \beta \text {, } \\
\qquad C(\beta)=\left[C\left(\vec{\varphi}_{1}\right), \ldots, C\left(\vec{\varphi}_{R}\right)\right]^{T}\end{array}$ \\
\hline$\delta_{+}^{K}$ & Space of $K \times K$ symmetric positive semi-definite matrices \\
\hline $\mathcal{X}$ & Input space \\
\hline $\mathcal{K}$ & Kernel machine \\
\hline $\mathcal{H}$ & Reproducing Kernel Hilbert Space (RKHS) associated with the kernel machine $\mathcal{K}$ \\
\hline$\Gamma$ & $\begin{array}{l}\text { A nonlinear map, } \Gamma: \mathcal{X} \rightarrow \mathcal{H} \text {, from } \mathcal{X} \text { to the high-dimensional Hilbert space } \mathcal{H} \\
\text { associated with } \mathcal{K}\end{array}$ \\
\hline$f_{d}(x, y)$ & L2 distance in $\mathcal{H}, f_{d}(x, y)=\|x-y\|_{2}^{2}$ \\
\hline$f_{k}^{\mathcal{C}}$ & $\begin{array}{l}\text { Probabilistic classifier function, } f_{k}^{\mathcal{C}}: \mathcal{S} \rightarrow[0,1] \text {, where } \\
\qquad \mathcal{S}=\bigcup_{\vec{\varphi}_{r} \in \beta}\left\{\varphi_{r, i} \mid \varphi_{r, i} \in \vec{\varphi}_{r}\right\}, \sum_{k=1}^{K} f_{k}^{\mathcal{C}}\left(\varphi_{r, i}\right)=1\end{array}$ \\
\hline$v_{k}\left(\varphi_{r, i}\right)$ & $\begin{array}{l}\text { Parameter, associated with a particular } \varphi_{r, i} \text { and } k \text {-th class } C_{k}, \\
\quad v_{k}\left(\varphi_{r, i}\right)=\frac{1}{\pi}\left(\varphi_{r, i}\right) .\end{array}$ \\
\hline$v_{k}\left(\vec{\varphi}_{r}\right)$ & $\begin{array}{l}\text { Collection of } L \text { parameters, } v_{k}\left(\vec{\varphi}_{r}\right)=\left[v_{k}\left(\varphi_{r, 1}\right), \ldots, v_{k}\left(\varphi_{r, L}\right)\right] \text {, where } \\
\quad \sum_{i=1}^{L} v_{k}\left(\varphi_{r, i}\right)=1\end{array}$ \\
\hline$\phi_{k}\left(\vec{\varphi}_{r}\right)$ & $\begin{array}{l}\text { Nonlinear map in } \mathcal{H} \text {, defined for a stabilized sequence } \vec{\varphi}_{r} \text { as } \\
\phi_{k}\left(\vec{\varphi}_{r}\right)=\left(\nu_{k}\left(\vec{\varphi}_{r}\right)\right)^{T} f_{k}^{\mathcal{C}}\left(\vec{\varphi}_{r}\right) \text {, where } \nu_{k}\left(\varphi_{r, i}\right)=\frac{1}{\pi}\left(\varphi_{r, i}\right) \text {, and } \\
\quad f_{k}^{\mathcal{C}}\left(\varphi_{r, i}\right) \in[0,1] \text { outputs a probability }\end{array}$ \\
\hline$\iota(\cdot)$ & Function, returns an inner product \\
\hline$Z$ & A parameter of procedure $\mathcal{P}_{S}$ \\
\hline$B$ & A parameter of procedure $\mathcal{P}_{S}$ \\
\hline$\ell_{k}\left(\vec{\varphi}_{r}\right)$ & A parameter of algorithm $\mathcal{A}_{C}$ \\
\hline$\xi\left(\vec{\varphi}_{r}\right)$ & A parameter of algorithm $\mathcal{A}_{C}$ \\
\hline
\end{tabular}

\section{References}

1. Biamonte, J., et al.: Quantum machine learning. Nature 549, 195-202 (2017)

2. LeCun, Y., Bengio, Y., Hinton, G.: Deep learning. Nature 521, 436-444 (2014)

3. Goodfellow, I., Bengio, Y., Courville, A.: Deep Learning. MIT Press, Cambridge (2016)

4. Debnath, S., et al.: Demonstration of a small programmable quantum computer with atomic qubits. Nature 536, 63-66 (2016)

5. Monz, T., et al.: Realization of a scalable Shor algorithm. Science 351, 1068-1070 (2016)

6. Barends, R., et al.: Superconducting quantum circuits at the surface code threshold for fault tolerance. Nature 508, 500-503 (2014)

7. Kielpinski, D., Monroe, C., Wineland, D.J.: Architecture for a large-scale ion-trap quantum computer. Nature 417, 709-711 (2002) 
8. Ofek, N., et al.: Extending the lifetime of a quantum bit with error correction in superconducting circuits. Nature 536, 441-445 (2016)

9. Farhi, E., Goldstone, J., Gutmann, S.: A quantum approximate optimization algorithm (2014). arXiv:1411.4028

10. Farhi, E., Goldstone, J., Gutmann, S., Neven, H.: Quantum algorithms for fixed qubit architectures (2017). arXiv:1703.06199v1

11. Farhi, E., Neven, H.: Classification with quantum neural networks on near term processors (2018). arXiv: $1802.06002 \mathrm{v} 1$

12. Farhi, E., Goldstone, J., Gutmann, S.: A quantum approximate optimization algorithm applied to a bounded occurrence constraint problem (2014). arXiv:1412.6062

13. Rebentrost, P., Mohseni, M., Lloyd, S.: Quantum support vector machine for big data classification. Phys. Rev. Lett. 113, 130503 (2014)

14. Lloyd, S.: The Universe as Quantum Computer, A Computable Universe: Understanding and exploring Nature as computation, H. Zenil (ed.) World Scientific, Singapore (2012); (2013). arXiv:1312.4455v1

15. Lloyd, S., Mohseni, M., Rebentrost, P.: Quantum algorithms for supervised and unsupervised machine learning (2013) arXiv:1307.0411v2

16. Lloyd, S., Garnerone, S., Zanardi, P.: Quantum algorithms for topological and geometric analysis of data. Nat. Commun. 7 (2016) arXiv:1408.3106

17. Lloyd, S., Shapiro, J.H., Wong, F.N.C., Kumar, P., Shahriar, S.M., Yuen, H.P.: Infrastructure for the quantum Internet. ACM SIGCOMM Comput. Commun. Rev. 34, 9-20 (2004)

18. Lloyd, S., Mohseni, M., Rebentrost, P.: Quantum principal component analysis. Nat. Phys. 10, 631 (2014)

19. Gyongyosi, L., Imre, S., Nguyen, H.V.: A survey on quantum channel capacities. IEEE Commun. Surv. Tutor. 99, 1 (2018). https://doi.org/10.1109/COMST.2017.2786748

20. Schuld, M., Sinayskiy, I., Petruccione, F.: An introduction to quantum machine learning. Contemp. Phys. 56, 172-185 (2015). arXiv: 1409.3097

21. Van Meter, R.: Quantum Networking. Wiley (2014). ISBN: 1118648927,9781118648926

22. Imre, S., Gyongyosi, L.: Advanced Quantum Communications-An Engineering Approach. WileyIEEE Press, New Jersey (2012)

23. Pirandola, S., Laurenza, R., Ottaviani, C., Banchi, L.: Fundamental limits of repeaterless quantum communications. Nat. Commun. 8, 15043 (2017). https://doi.org/10.1038/ncomms 15043

24. Pirandola, S., Braunstein, S.L., Laurenza, R., Ottaviani, C., Cope, T.P.W., Spedalieri, G., Banchi, L.: Theory of channel simulation and bounds for private communication. Quant. Sci. Technol. 3, 035009 (2018)

25. Pirandola, S.: Capacities of repeater-assisted quantum communications (2016). arXiv:1601.00966

26. Pirandola, S.: End-to-end capacities of a quantum communication network. Commun. Phys. 2, 51 (2019)

27. Petz, D.: Quantum Information Theory and Quantum Statistics. Hiv: 6. Springer, Heidelberg (2008)

28. Preskill, J.: Quantum computing in the NISQ era and beyond. Quantum 2, 79 (2018)

29. Harrow, A.W., Montanaro, A.: Quantum computational supremacy. Nature 549, 203-209 (2017)

30. Aaronson, S., Chen, L.: Complexity-theoretic foundations of quantum supremacy experiments. In: Proceedings of the 32nd Computational Complexity Conference (CCC '17), pp 22:1-22:67 (2017)

31. Zhou, X., Leung, D.W., Chuang, I.L.: Methodology for quantum logic gate construction. Phys. Rev. A 62, 052316 (2000)

32. Gottesman, D., Chuang, I.L.: Quantum teleportation is a universal computational primitive. Nature 402, 390-393 (1999)

33. Amy, M., Maslov, D., Mosca, M., Roetteler, M.: A meet-in-the middle algorithm for fast synthesis of depth-optimal quantum circuits. IEEE Trans. Comput. Aid. Des. Integr. Circuits Syst. 32(6), 818-830 (2013)

34. Paler, A., Polian, I., Nemoto, K., Devitt, S.J.: Fault-tolerant, high level quantum circuits: form, compilation and description. Quant. Sci. Technol. 2(2), 025003 (2017)

35. Brandao, F.G.S.L., Broughton, M., Farhi, E., Gutmann, S., Neven, H.: For fixed control parameters the quantum approximate optimization algorithm's objective function value concentrates for typical instances (2018). arXiv:1812.04170

36. Zhou, L., Wang, S.-T., Choi, S., Pichler, H., Lukin, M.D.: Quantum approximate optimization algorithm: performance, mechanism, and implementation on near-term devices (2018) arXiv:1812.01041 (2018) 
37. Lechner, W.: Quantum approximate optimization with parallelizable gates (2018). arXiv:1802.01157v2

38. Crooks, G.E.: Performance of the quantum approximate optimization algorithm on the maximum cut problem (2018). arXiv:1811.08419

39. Ho, W.W., Jonay, C., Hsieh, T.H.: Ultrafast state preparation via the quantum approximate optimization algorithm with long range interactions (2018). arXiv:1810.04817

40. Song, C., et al.: 10-qubit entanglement and parallel logic operations with a superconducting circuit. Phys. Rev. Lett. 119(18), 180511 (2017)

41. Chawky, B.S., Elons, A.S., Ali, A., Shedeed, H.A.: A study of action recognition problems: dataset and architectures perspectives. In: Hassanien, A.E. Oliva, D.A. (eds.) Advances in Soft Computing and Machine Learning in Image Processing, Studies in Computational Intelligence, vol. 730 (2018)

42. Miao, J., Xu, X., Xing, X., Tao, D.: Manifold regularized slow feature analysis for dynamic texture recognition (2017). arXiv:1706.03015v1

43. Cherian, A., Gould, S.: Second-order temporal pooling for action recognition (2017). arXiv: $1704.06925 \mathrm{v} 1$

44. Mika, S., Scholkopf, B., Smola, A., Muller, K.R., Scholz, M., Ratsch, G.: Kernel pca and de-noising in feature spaces. In: Advances in Neural Information Processing Systems, vol. 11, pp. 536-542. MIT Press (1999)

45. Shawe-Taylor, J., Cristianini, N.: Kernel Methods for Pattern Analysis. Cambridge University Press, Cambridge (2004)

46. Liu, W., Principe, J., Haykin, S.: Kernel Adaptive Filtering: A Comprehensive Introduction. Wiley, Hoboken (2010)

47. Wiskott, L., Sejnowski, T.J.: Slow feature analysis: Unsupervised learning of invariances. Neural Comput. 14, 715-770 (2002)

Publisher's Note Springer Nature remains neutral with regard to jurisdictional claims in published maps and institutional affiliations. 